\title{
Urinary lithiasis: diagnostic investigation
}

\author{
Ernesto Reggio ${ }^{1}$ \\ Alexandre Danilovic ${ }^{1}$ \\ Francisco Tustumi ${ }^{1}$ \\ Wanderley Marques Bernardo²
}

Contact:wmbernardo@usp.br

1. Sociedade Brasileira de Urologia, Rio de Janeiro, RJ, Brasil 2. Coordenador do Programa Diretrizes da Associação Médica Brasileira, São Paulo, SP, Brasil

Question: What evaluation is needed for better diagnosis and planning of patients with complex renal lithiasis who must be submitted to surgical treatment, such as percutaneous kidney lithotripsy?

Answer: For the best therapeutic planning, patients who are candidates to percutaneous kidney lithotripsy must be submitted to computed tomography for detailing of the anatomical structures, the collecting system, and the stone, in order to plan the puncture pathway. The use of intravenous contrast-enhanced computed tomography should be considered in complex anatomical situations, such as malformations or previous kidney surgery, in which it is desirable to know the anatomy of the renal collecting system. Alternatively, excretory urography can be used to evaluate the anatomy of the renal collecting system, but it does not replace the computed tomography since it does not allow the visualization of neighboring organs and their relationships with the kidney. ${ }^{1}$

\section{REFERENCE}

1. Reggio E, Danilovic A, Tustumi F, Bernardo WM. Urinary lithiasis: diagnostic investigation. Rev Assoc Med Bras. 2019; 65(8):1038-1038. 